\title{
Fermionic Condensate on Finite Radius Cones
}

\author{
A.A. Hovhannisyan \\ Institute of Applied Problems of Physics NAS RA 25 Hr. Nersessian Str., \\ 0014 Yerevan, Armenia \\ https://doi.org/10.52853/18291171-2021.14.1-1 \\ E-mail:arshakhov@gmail.com \\ Received 15 February 2021
}

\begin{abstract}
The fermionic condensate is investigated for a field localized on a finite radius 2dimensional cone in the presence of a magnetic flux threading the cone apex. On the edge of the cone a boundary condition is imposed that differs from the MIT bag boundary condition, most frequently used for the confinement of fermions. The fermionic condensate is decomposed into the boundary-free and edge-induced contributions. Both these parts are periodic functions of the magnetic flux with the period equal to the flux quantum.
\end{abstract}

Keywords: fermionic condensate, conical geometry, graphene

\section{Introduction}

In a number of planar condensed matter systems the long wavelength excitations of the electronic subsystem are described by a $(2+1)$ - dimensional fermionic field theory. Examples of this kind of systems are graphene, Weyl semimetals and topological insulators. In the corresponding Dirac equation the Fermi velocity appears instead of the speed of light (see, for example, [1, 2]). The effective field theory description of the condensed matter systems provides an interesting possibility to investigate the effects of topology and boundaries on the properties of the quantum vacuum. One-loop quantum effects in graphene made cylindrical and toroidal nanotubes have been studied in [3]-[8]. A more complicated geometry of hemisphere capped tubes is considered in [9]. The ground state charge and current densities of a fermionic field on curved graphene tubes with locally anti-de Sitter geometry were discussed in [10,11].

Another class of topologically nontrivial structures correspond to graphene nanocones (for the electronic properties see [13]-[16]). They are obtained from the graphene sheet cutting one or more sectors with angle $\pi / 6$. For the number of the removed sectors $n_{c}=1,2, \ldots, 5$ the opening angle of the cone is given by the formula $2 \pi\left(1-n_{c} / 6\right)$. Graphene cones with these angles have been observed in experiments [17]. The vacuum polarization in graphene with a conical topological defect is investigated in [19]-[27] within the framework of a long-wavelength continuum model. In the main part of those papers for cones with edges the MIT bag boundary condition was imposed on the fermionic field. In the present paper we consider the ground state fermionic condensate on a finite radius cone for a different type of boundary condition on the edge of the cone. 
The organization of the paper is as follows. In the next section the geometry is described and the mode functions of the fermionic field are given. The fermionic condensate is investigated analytically and numerically in section 3 . The main results are summarized in section 4 .

\section{Geometry and the modes}

We consider a charged spinor field $\psi$ on two-dimensional conical space. In the irreducible representation it has two components and the corresponding dynamics is described by the Dirac equation

$$
i \gamma^{\mu}\left(\partial_{\mu}+\Gamma_{\mu}+i e A_{\mu}\right) \psi-m \psi=0
$$

where $\Gamma_{\mu}$ is the spin connection and $A_{\mu}$ is the vector potential for a classical gauge field. The spacetime geometry under consideration is given by the line element

$$
d s^{2}=g_{\mu \nu} d x^{\mu} d x^{v}=d t^{2}-d r^{2}-r^{2} d \phi^{2}
$$

with the radial coordinate. The angular coordinate takes the values in the range $0 \leq \phi \leq \phi_{0}$, where $2 \pi-\phi_{0}$ is the measure of planar angle deficit for the conical space. For $r \neq 0$ the geometry (2) is flat and the curvature tensor has delta type singularity at $r=0$ for $\phi_{0} \neq 0$. In (1) the $2 \times 2$ Dirac matrices are given in polar coordinates $(r, \phi)$. If $\gamma^{b}, b=0,1,2$, are the corresponding matrices in Cartesian coordinates, then we have the relation $\gamma^{\mu}=e_{(b)}^{\mu} \gamma^{(b)}$ where $e_{(b)}^{\mu}$ are the tetrad fields. In the discussion below we will take $\gamma^{(0)}=\sigma_{3}, \gamma^{(1)}=i \sigma_{1}, \gamma^{(2)}=i \sigma_{2}$, where $\sigma_{1}, \sigma_{2}, \sigma_{3}$ are the standard Pauli matrices. It is convenient to use the representation $e_{(0)}^{\mu}=(1,0,0)$ and

$$
\begin{aligned}
& e_{(1)}^{\mu}=(0, \cos (q \phi),-\sin (q \phi) / r), \\
& e_{(2)}^{\mu}=(0, \sin (q \phi), \cos (q \phi) / r),
\end{aligned}
$$

with $q=2 \pi / \phi_{0}$. We assume that the cone has a finite radius $a$. On the edge $r=a$ the field is constrained by the boundary condition

$$
\left(1-i n_{\mu} \gamma^{\mu}\right) \psi(x)=0
$$

where $n_{\mu}=\delta_{\mu}^{1}$ is the inward pointing unit vector normal to the boundary. In the discussion below the magnetic field configuration will correspond to an infinitely thin magnetic flux located at the apex of the cone. The vector potential for that configuration is given by $A_{\mu}=(0,0, A)$ in the region $r>0$. The component $A_{3}=A$ is related to the magnetic flux $\Phi$ by the formula $A=-\Phi / \phi_{0}$. The boundary condition (4) differs from the MIT bag boundary condition, widely discussed in the literature, by the sign of the term containing the normal to the edge. The 
fermionic condensate for the bag boundary condition has been investigated in [21]. The vacuum expectation values of the fermionic charge and current densities in conical rings, with boundary conditions that include also the condition (4), have been investigated recently in [27].

The fermion condensate in the vacuum state $|0\rangle$ is defined as the vacuum expectation value $\langle 0|\bar{\psi} \psi| 0\rangle=\langle\bar{\psi} \psi\rangle$, where $\bar{\psi}=\psi^{\dagger} \gamma^{0}$ is Dirac adjoint and the dagger denotes Hermitian conjugation. For the evaluation of the FC we need the complete set of fermionic mode functions $\psi_{\sigma}^{(\kappa)}(x)$ that obey the boundary condition (4). Here $\kappa=+$ and $\kappa=-$ correspond to the positive and negative energy functions and the collective index $\sigma$ stands for the set of quantum numbers specifying the solutions. The mode functions are given by

$$
\psi_{\sigma}^{(\kappa)}(x)=C_{\kappa} e^{i q j \phi-\kappa i E t}\left(\begin{array}{c}
J_{\beta_{j}}(\gamma, r) e^{-i q \phi / 2} \\
\varepsilon_{j} \gamma \frac{e^{i q \phi / 2}}{\kappa E+m} J_{\beta_{j}+\varepsilon_{j}}(\gamma, r)
\end{array}\right),
$$

where $J_{v}(x)$ is the Bessel function, $j= \pm 1 / 2, \pm 3 / 2, \ldots, \varepsilon_{j}=1$ for $j>-\alpha$ and $\varepsilon_{j}=-1$ for $j<-\alpha$, with $\alpha=e A / q=-e \Phi /(2 \pi)$, and

$$
\beta_{j}=q|j+\alpha|-\varepsilon_{j} / 2
$$

From the boundary condition (4) it follows that the allowed values of the radial quantum number $\gamma$ are the roots of the equation

$$
\varepsilon_{j} \gamma J_{\beta_{j}+\varepsilon_{j}}(\gamma a)+\left(\kappa \sqrt{\gamma^{2}+m^{2}}+m\right) J_{\beta_{j}}(\gamma a)=0 .
$$

The spectrum of $\gamma$ is discrete and the normalization coefficient in (5) is determined from the condition

$$
\int_{0}^{a} d r \int_{0}^{\phi_{0}} d \phi r \psi_{\sigma}^{(\kappa) \dagger}(x) \psi_{\sigma}^{(\kappa)}(x)=1 .
$$

The radial integral in the latter relation is evaluated by using the standard integral for the square of the Bessel function (see, for example, [28]).

\section{Fermionic Condensate}

Having specified the mode functions and the eigenvalues of the radial quantum number $\gamma$, the fermionic condensate is evaluated by using the formula $\langle\bar{\psi} \psi\rangle=\sum_{\kappa, j, \gamma} \kappa \bar{\psi}_{\sigma}^{(\kappa)} \psi_{\sigma}^{(\kappa)} / 2$. The summation over $\gamma$ goes over the roots of the equation (7). For the summation of the corresponding series we can use the generalized Abel-Plana formula [29]. The details of the evaluation is similar to that given in [21] and we will give the final result. details. The fermionic condensate is presented in the form of the sum of two contributions: 


$$
\langle\bar{\psi} \psi\rangle=\langle\bar{\psi} \psi\rangle_{0}+\langle\bar{\psi} \psi\rangle_{e}
$$

The first term in the right-hand side, $\langle\bar{\psi} \psi\rangle_{0}$, is the fermionic condensate in a conical space without edge $(0 \leq r<\infty)$ and the second term is the part induced by the edge at $r=a$. The renormalized value of the first part is given in [21]:

$$
\begin{aligned}
& \langle\bar{\psi} \psi\rangle_{0}=-\frac{m}{2 \pi r}\left[\sum_{l=1}^{[q / 2]} \frac{(-1)^{l} \cot (\pi l / q)}{e^{2 m r \sin (\pi l / q)} \cos 2 \pi l \alpha_{0}+}\right. \\
& \left.+\frac{q}{\pi} \sum_{\delta= \pm 1} \cos \left[q \pi\left(1+\delta \alpha_{0}\right)\right] \times \int_{0}^{\infty} d y \frac{e^{-2 m r \cosh y}}{\cosh y} \frac{\sinh y \cdot \sinh \left[q\left(1-2 \delta \alpha_{0}\right) y\right]}{\cosh 2 q y-\cos q \pi}\right]
\end{aligned}
$$

where $[q / 2]$ is the integer part of $q / 2$. For $1 \leq q<2$ the term with the summation over $l$ is absent. In (10) the parameter $\alpha_{0}$ is defined by the relation $\alpha=\alpha_{0}+n_{0}$, where $n_{0}$ is an integer and $\left|\alpha_{0}\right|<1 / 2$. The contribution induced by the presence of the edge is presented in the form

$$
\begin{aligned}
& \langle\bar{\psi} \psi\rangle_{e}=\frac{q}{2 \pi^{2}} \sum_{j} \int_{m}^{\infty} d x \operatorname{Re}\left[\frac{x K_{\beta_{j}+\varepsilon_{j}}(x a)+\left(m+i \sqrt{x^{2}-m^{2}}\right) K_{\beta_{j}}(x a)}{x I_{\beta_{j}+\varepsilon_{j}}(x a)-\left(m+i \sqrt{x^{2}-m^{2}}\right) I_{\beta_{j}}(x a)} \times\right. \\
& \left.\times\left(\frac{m}{\sqrt{x^{2}-m^{2}}}-i\right) I_{\beta_{j}+\varepsilon_{j}}^{2}(x r)-\left(\frac{m}{\sqrt{x^{2}-m^{2}}}+i\right) I_{\beta_{j}}^{2}(x r)\right]
\end{aligned}
$$

where $I_{v}(x)$ and $K_{v}(x)$ are the modified Bessel functions. By redefining the summation variable over $j$ we see that this contribution does not depend on the integer part $n_{0}$ of $\alpha$. Combining with (10) we conclude that the fermionic condensate is a periodic function of the magnetic flux with the period equal to the flux quantum $2 \pi / e$.

For a massless fermionic field the part $\langle\bar{\psi} \psi\rangle_{0}$ vanishes and the edge-induced part is transformed to

$$
\langle\bar{\psi} \psi\rangle_{e}=\frac{q}{2 \pi^{2} a} \sum_{j} \int_{0}^{\infty} d x \frac{I_{\beta_{j}+\varepsilon_{j}}^{2}(x r)+I_{\beta_{j}}^{2}(x r)}{I_{\beta_{j}+\varepsilon_{j}}^{2}(x a)+I_{\beta_{j}}^{2}(x a)} .
$$

The latter is always positive. As seen from (12), the fermionic condensate diverges on the edge. The leading term in the expansion near the edge is found in a way similar to that described in [21] and $\langle\bar{\psi} \psi\rangle_{e} \approx 1 /\left(8 \pi(a-r)^{2}\right)$. Near the cone apex, $r \rightarrow 0$, the edge-induced contribution behaves as $r^{q\left(1-2\left|\alpha_{0}\right|\right)-1}$. In figure 1 we have plotted the fermionic condensate for a massless filed as a function of $r / a$ for different values of the parameter $q$ (the numbers near the curves) and for $\alpha_{0}=1 / 4$. 


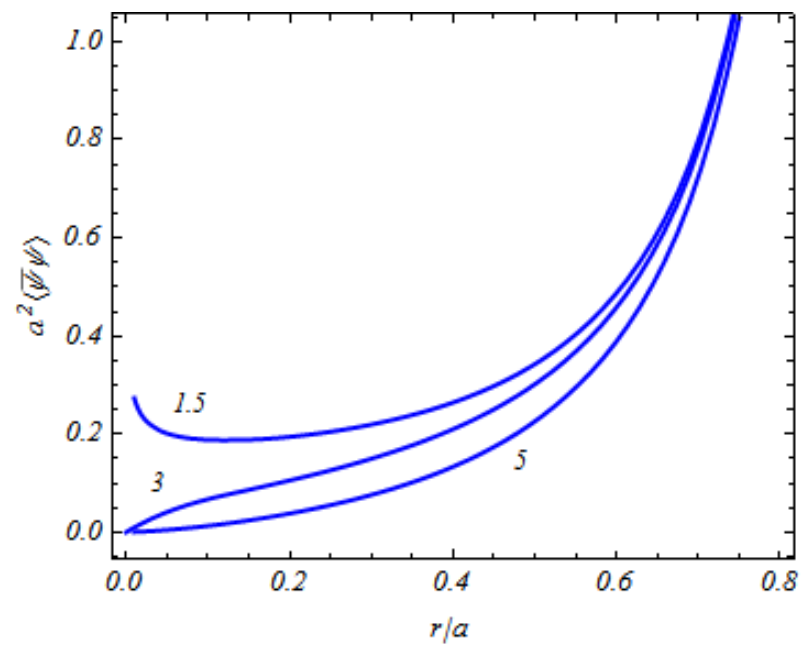

Figure 1:The fermionic condensate for a massless field as a function of the radial coordinate for $\mathrm{q}=1.5,3,5$ and $\alpha_{0}=1 / 4$.

In figure 2, the fermionic condensate is plotted versus the parameter $\alpha_{0}$ for a massless field and for fixed value of the radial coordinate $r=a / 2$. The numbers near the curves are the values of the parameter $q$.

The dependence of the fermionic condensate on the mass of the field is displayed in figure figure 3 for $\alpha_{0}=1 / 4, r=a / 2$, and for different values of $q$ (numbers near curves). As it is seen from the graphs, the dependence on the mass is not monotonic. For large values of the mass the condensate tends to zero.

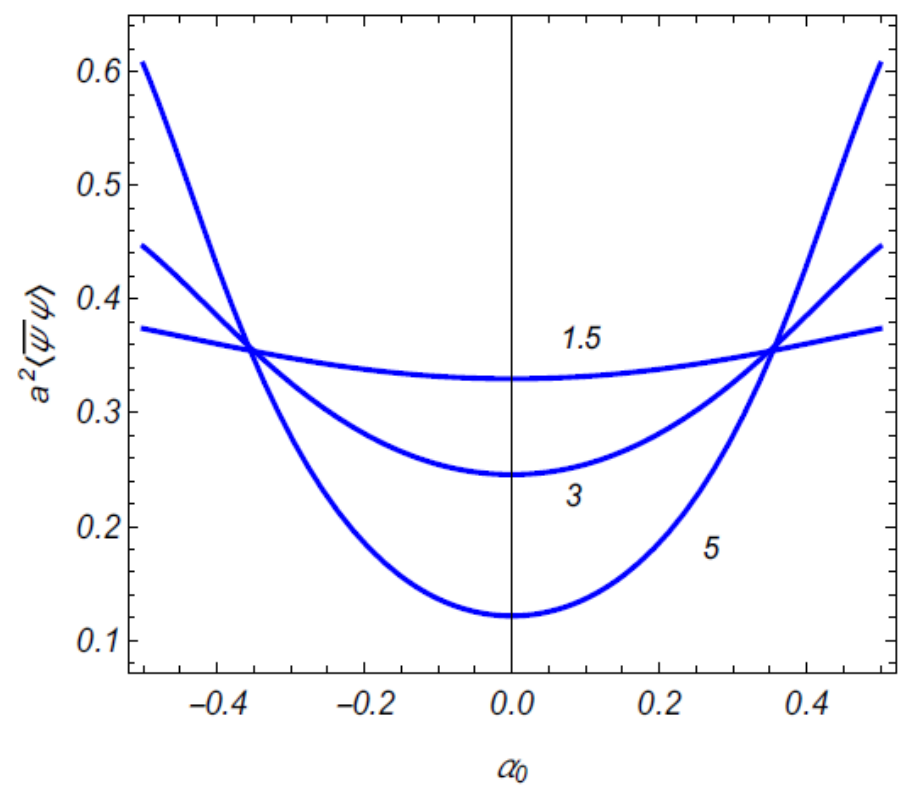

Figure 2: The fermionic condensate for a massless field versus the parameter $\alpha_{0}$ for $q=1.5,3,5$ and $r=a / 2$. 


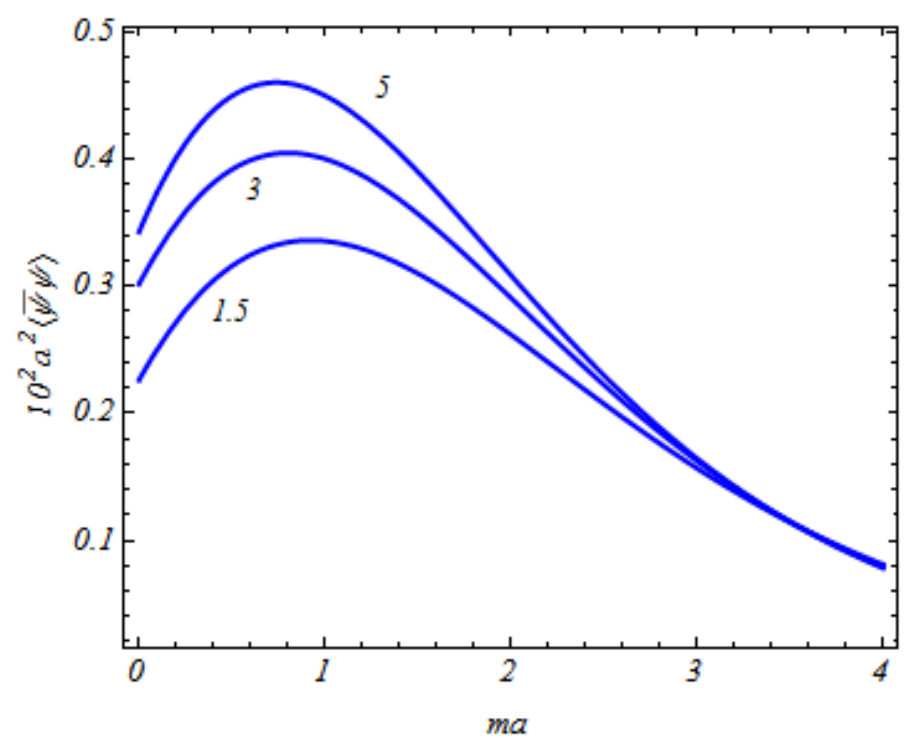

Figure 3: The fermionic condensate versus the parameter $m$ for $q=$ $1.5,3,5, r=a / 2$ and $\alpha_{0}=1 / 4$.

\section{Conclusion}

We have investigated the fermionic condensate for a field localized on a finite radius cone with an arbitrary opening angle. On the edge of the cone the field obeys the boundary condition (4). The corresponding problem for the MIT bag boundary condition has been considered in [21]. The boundary condition (4) differs from the bag boundary condition by the sign of the term containing the normal to the edge. The complete set of fermionic mode functions are given by (5). The eigenvalues of the radial quantum number are quiantized by the boundary condition on the edge and are roots of the equation (7). The mode sum for the fermion condensate containes series over those roots. For the summation of the series the generalized Abel-Plana was used. That allowed us to extract from the condensate the part corresponding to a conical geometry in the absence of the edge and to obtain for the edge-induced contribution in the for well adapted for numerical investigations. The fermionic condensate is an even periodic function of the magnetic flux with the period of the flux quantum.

\section{Acknowledgments}

The author is grateful to Professor A.A. Saharian for the help during the work on the paper.

\section{References}

[1] V.P. Gusynin, S.G. Sharapov, J.P. Carbotte, Int. J. Mod. Phys. B 21, 4611 (2007).

[2] A.H. Castro Neto, F. Guinea, N.M.R. Peres, K.S. Novoselov, A.K. Geim, Rev. Mod. Phys. 81, 109 (2009). 
[3] S. Bellucci, A.A. Saharian, Phys. Rev. D 79, 085019 (2009).

[4] S. Bellucci, A.A. Saharian, Phys. Rev. D 80, 105003 (2009).

[5] S. Bellucci, A.A. Saharian, V.M. Bardeghyan, Phys. Rev. D 82, 065011 (2010).

[6] E. Elizalde, S.D. Odintsov, A.A. Saharian, Phys. Rev. D 83, 105023 (2011).

[7] S. Bellucci, A.A. Saharian, Phys. Rev. D 87, 025005 (2013).

[8] S. Bellucci, E.R. Bezerra de Mello, A.A. Saharian, Phys. Rev. D 89, 085002 (2014).

[9] E.R. Bezerra de Mello, A.A. Saharian, Int. J. Theor. Phys. 55, 1167 (2016).

[10] S. Bellucci, A.A. Saharian, V. Vardanyan, Phys. Rev. D 96, 065025 (2017).

[11] S. Bellucci, A.A. Saharian, D. H. Simonyan, V. Vardanyan, Phys. Rev. D 98, 085020 (2018).

[12] S. Bellucci, A.A. Saharian, D. H. Simonyan, H. G. Sargsyan, V. Vardanyan, Phys. Rev. D. 101, 045020 (2020).

[13] P.E. Lammert, V.H. Crespi, Phys. Rev. Lett. 85, 5190 (2000).

[14] A. Cortijo, M.A.H. Vozmediano, Nucl. Phys. B 763, 293 (2007).

[15] Yu.A. Sitenko, N.D. Vlasii, Nucl. Phys. B 787, 241 (2007).

[16] C. Furtado, F. Moraes, A.M.M. Carvalho, Phys. Lett. A 372, 5368 (2008).

[17] A. Krishnan, et al, Nature 388, 451 (1997).

[18] S.N. Naess, A. Elgsaeter, G. Helgesen, K.D. Knudsen, Sci. Technol. Adv. Mater. 10, 065002 (2009).

[19] Yu.A. Sitenko, N.D. Vlasii, Low Temp. Phys. 34, 826 (2008).

[20] E. R. Bezerra de Mello, V. Bezerra, A.A. Saharian, V.M. Bardeghyan, Phys. Rev. D 82, 085033 (2010).

[21] S. Bellucci, E.R. Bezerra de Mello, A.A. Saharian, Phys. Rev. D 83, 085017 (2011).

[22] E.R. Bezerra de Mello, F. Moraes, A.A. Saharian, Phys. Rev. D 85, 045016 (2012).

[23] S. Bellucci, E.R. Bezerra de Mello, E. Bragan, ca, A.A. Saharian, Eur. Phys. J. C 76, 359 (2016).

[24] Yu.A. Sitenko, V.M. Gorkavenko, Low Temp. Phys. 44, 1261 (2018).

[25] Yu.A. Sitenko, V.M. Gorkavenko, Phys. Rev. D 100, 085011 (2019).

[26] A.A. Saharian, E.R. Bezerra de Mello, A.A. Saharyan, Phys. Rev. D 100, 105014 (2019).

[27] S. Bellucci, I. Brevik, A.A. Saharian, H.G. Sargsyan, Eur. Phys. J. C 80, 281 (2020).

[28] A. P. Prudnikov, Yu. A. Brychkov, and O. I. Marichev, Integrals and Series (Gordon and Breach, New York, 1986), Vol. 2.

[29] A.A. Saharian, The Generalized Abel-Plana Formula with Applications to Bessel Functions and Casimir Effect (Yerevan State University Publishing House, Yerevan, 2008); Report No. ICTP/2007/082; arXiv:0708.1187. 